\title{
A NON-OVERLAPPING DOMAIN DECOMPOSITION METHOD FOR CONTINUOUS-PRESSURE MIXED FINITE ELEMENT APPROXIMATIONS OF THE STOKES PROBLEM*,**
}

\author{
Hani Benhassine ${ }^{1,2}$ and Abderrahmane Bendali ${ }^{2}$
}

\begin{abstract}
This study is mainly dedicated to the development and analysis of non-overlapping domain decomposition methods for solving continuous-pressure finite element formulations of the Stokes problem. These methods have the following special features. By keeping the equations and unknowns unchanged at the cross points, that is, points shared by more than two subdomains, one can interpret them as iterative solvers of the actual discrete problem directly issued from the finite element scheme. In this way, the good stability properties of continuous-pressure mixed finite element approximations of the Stokes system are preserved. Estimates ensuring that each iteration can be performed in a stable way as well as a proof of the convergence of the iterative process provide a theoretical background for the application of the related solving procedure. Finally some numerical experiments are given to demonstrate the effectiveness of the approach, and particularly to compare its efficiency with an adaptation to this framework of a standard FETI-DP method.
\end{abstract}

Mathematics Subject Classification. 76D07,65N55, 65N30.

Received August 20, 2009. Revised June 20, 2010.

Published online November 30, 2010.

\section{INTRODUCTION}

This paper deals with a non-overlapping domain decomposition method (DDM) for solving the continuouspressure mixed finite element formulations of the Stokes equations. The guideline is the strategy initiated by Lions [27] for second order elliptic boundary values problems, called later Robin-Robin DDMs. It seems that it is more appropriate to refer to these methods as mixed non-overlapping DDMs in the discrete framework [20]. The main principle is to decompose the computational domain into several non-overlapping subdomains and to express the natural matching of the trace of the solution and its normal derivative at the interfaces in terms

\footnotetext{
Keywords and phrases. Stokes equations, incompressible fluids, domain decomposition methods, non-overlapping domain decomposition methods, FETI-DP methods, cross points.

* The research of the first author was supported by a grant within the framework of the French-Algerian program PROFAS. He is indebted to the Department of Engineering Mathematics and to the Toulouse Mathematics Institute for their kind hospitality during the achievement of this work.

** The authors would like to thank the anonymous reviewers for their thorough reading and their comments which contribute to improve the final appearance of this paper.

1 Département de Mathématiques, Université de Jijel, BP 98 Aouled Aissa, 18000 Jijel, Algeria.

2 Université de Toulouse, Institut Mathématique de Toulouse, Département de Génie Mathématique, INSA de Toulouse, 135 avenue de Rangueil, 31077 Toulouse Cedex 4, France. h.benhassine@gmail.com; abendali@insa-toulouse.fr
} 


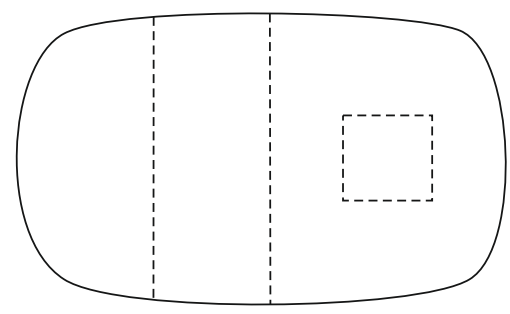

FigurE 1. A decomposition without cross points.

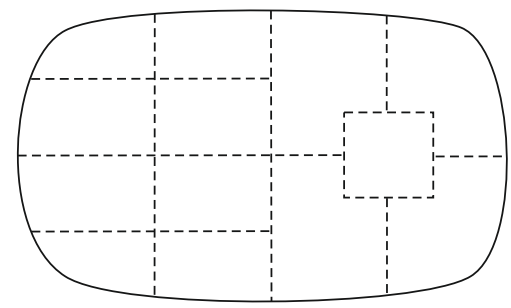

Figure 2. A decomposition with cross points.

of two Robin conditions. For the Stokes system, the Robin condition is obtained by a combination of the velocity and the normal stress vector. This has the advantage of ensuring that each iteration can be performed in a stable way independently of the mesh size. In particular, this way to proceed avoids being faced with the so-called 'floating subdomains' which prevent the related local problems from being safely solved.

First theoretical well-posedness and convergence results for the Robin-Robin DDM, applied to the 'continuous' Stokes/Oseen problem (i.e., in the Sobolev spaces setting), were established in [31]. As usual in such a kind of study, devoted to the theoretical analysis of the DDM without connection to some numerical scheme [13,27], the difficulties, related to the 'corners' where several subdomains meet, are avoided by assuming an extra non variational regularity on the normal derivatives (the normal stress vector here) at the subdomains interfaces. Unfortunately, this way to proceed cannot be reproduced at the discrete level when using a nodal finite element method. Actually, avoiding a correct handling of the matching at the 'corners' of the subdomains only postpones the difficulty that arises from the cross points in the discrete case. Figures 1 and 2 respectively give an idea of a decomposition without and with cross points. Indeed, such cross points may support one or more degrees of freedom for which the equivalent writing of the matching conditions, which is the basic feature of the Lions method, no longer remains valid.

In this study, we focus on a way of proceeding with a DDM involving cross points for the continuouspressure mixed finite element formulations of the Stokes problem, which can ensure the convergence and stability properties of this procedure at the theoretical level. The approach adopted here to overcome the difficulties related to cross points is that considered in references $[2,3,5,17]$. It consists in keeping unchanged each unknown and each equation of the finite element system related to a cross point. Moreover, as for the treatment of the Helmholtz equation, initiated by Després in [14], and adapted to this context in $[2,3,5]$, each local matrix is then augmented by a suitable interface mass matrix. Lagrange multipliers are introduced to enforce the matching of the Robin conditions at the nodes located at the interfaces except those corresponding to the cross points. As a result, one gets an augmented linear system which remains exactly equivalent to the global discrete Stokes problem. As this is characteristic of a DDM, the problem is reduced to an implicit linear system on the interface Lagrange multipliers where the matrix-vector product is obtained by solving local problems, each of them being related to a subdomain. For the present case, each matrix-vector product is performed by solving the local problems twice and, inserted between the two series of these solutions, an intermediate linear system related to the degree of freedom supported by the cross points. It is important to note that this way to proceed makes it possible to prescribe the zero mean-value condition for the pressure easily through an additional Lagrange multiplier which is joined to the degrees of freedom corresponding to the cross points.

To some extent, this procedure can be linked to the FETI Dual-Primal (FETI-DP) method introduced in [17] as an improvement on the standard FETI approach initiated in [16]. In the FETI-DP method also the degrees of freedom supported by a cross point remain shared by all the subdomains to which this cross point belongs (see, for example, [29]). The matching of the remaining degrees of freedom on subdomain interfaces is enforced by Lagrange multipliers. All the other degrees of freedom are removed via a Schur complement procedure and the resulting symmetric linear system is solved by an iterative solver. However, the FETI-DP method does not seem to have been used in the context of a continuous-pressure mixed finite element approximation 
of the Stokes problem, mainly because some additional treatment must be carried out for matching the pressure at the interfaces. However, to be able to compare the method which is designed in this work with a standard FETI approach, we show how to extend the latter to the continuous-pressure mixed finite element approximations of the Stokes system. The main ingredient is to deal in a formal way with the whole system as if it were a usual coercive elliptic problem. This way of proceeding remains questionable mainly because its validity and its stability cannot be based on a sound theoretical background.

Many works have been devoted to DDMs for saddle point problems in general and for the Stokes problem in particular. They are based on either overlapping [24,35] or non-overlapping Schwarz methods [1,9,19,25,30,33]. For instance, the Stokes problem was dealt with by a Robin-Robin method in Otto et al. [28,32], Chacón Rebollo and Chacón Vera [11,12] and Discacciati et al. [15]. However, in all these references, no specific attention was paid to the adaptations required for the cross points. Furthermore, in all these studies the finite element approximation of the pressure is assumed to be discontinuous through the interfaces of the subdomains. More recently, Vereecke et al. [37] (for incompressible and nearly incompressible problems), Li [26] and Kim and Lee [22] (for mortar finite element methods), used a FETI-DP method [17] for the Stokes equations to settle the problem resulting from the cross points. However, only discontinuous-pressure finite element methods were considered. Our aim in this study is to investigate such issues and to propose a suitable approach to them. Great attention is paid to the stability properties of the method. In particular, a strict control is carried out at the level where the local problems have to be solved as well as where the degrees of freedom supported by the cross points need to be determined. In particular, we carefully check a bound on the results in terms of the data using suitable norms. It is worth mentioning that this is in contrast with the standard FETI-DP method $[22,26,37]$, where the existence and the uniqueness of the solution to the local problems is ascertained from an algebraic argument only.

As mentioned above, the construction of the DDM and the theoretical study are conducted mainly for continuous-pressure finite element approximations of the Stokes problem. A similar, but simpler, construction leading to the same conclusions remains valid when using a discontinuous-pressure finite element method. The related adaptations will be indicated along the text.

This paper is organized as follows. Section 1 contains a brief introduction to the variational velocity-pressure formulation of the Stokes problem as well as a description of the additive Schwarz non-overlapping DDM that is considered. Theoretical stability estimates and convergence results are established in Section 2 . Some numerical experiments validating the theoretical conclusions and comparing its efficiency with an adaptation of the standard FETI-DP method to this framework are presented in Section 3.

\section{Mixed DDM FORMulation}

\subsection{Stokes equations}

We consider the Stokes equations in a bounded domain $\Omega$ of $\mathbb{R}^{n}(n=2,3)$, together with a non-slipping condition on the boundary $\partial \Omega$ of $\Omega$ and the incompressibility constraint (cf., e.g., [18])

$$
\left\{\begin{array}{rlrl}
-\nu \Delta \mathbf{u}+\nabla p & =\mathbf{f}, & & \text { on } \Omega, \\
\nabla \cdot \mathbf{u}=0, & & \text { on } \Omega, \\
\mathbf{u}= & 0, & & \text { on } \partial \Omega .
\end{array}\right.
$$

In system (1.1) the unknowns are $\mathbf{u} \in\left(H_{0}^{1}(\Omega)\right)^{n}$ the velocity and $p \in L^{2}(\Omega)$ the pressure. The data are respectively $\nu$ the inverse of the Reynolds number and $\mathbf{f} \in\left(L^{2}(\Omega)\right)^{n}$ the external forces per unit mass moving the fluid. The pressure is defined up to an additive constant which, as usual, is fixed by taking

$$
p \in L_{0}^{2}(\Omega)=\left\{q \in L^{2}(\Omega) ; \int_{\Omega} q \mathrm{~d} x=0\right\} .
$$




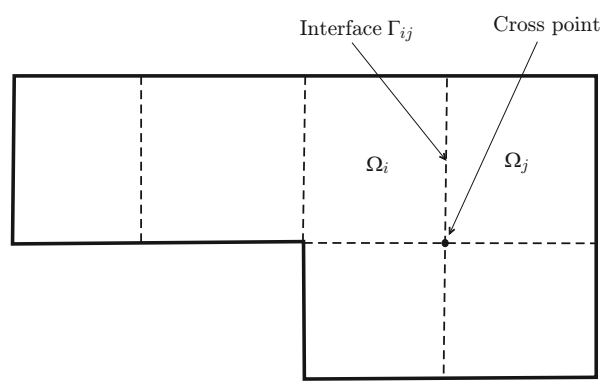

FIGURE 3. Non-overlapping decomposition of the domain $\Omega$ in six subdomains.

System (1.1) is solved using a nodal mixed finite element method. Let $\mathcal{T}^{h}$ be a mesh of $\Omega$, with fixed shape geometrical elements of diameter $\leq h$. We denote the velocity and the pressure finite element spaces by $X^{h} \subset\left(H_{0}^{1}(\Omega)\right)^{n}$ and $M^{h} \subset L^{2}(\Omega)$ respectively. The discrete variational formulation of the Stokes problem can then be set as follows:

$$
\begin{cases}\mathbf{u} \in X^{h}, p \in M_{0}^{h} & \\ a(\mathbf{u}, \mathbf{v})+b(\mathbf{v}, p)=(\mathbf{f}, \mathbf{v})_{\Omega}, & \forall \mathbf{v} \in X^{h}, \\ b(\mathbf{u}, q)=0, & \forall q \in M_{0}^{h},\end{cases}
$$

where $M_{0}^{h}$ is the subspace of functions in $M^{h}$ satisfying zero mean value condition (1.2) $\left(M_{0}^{h}=M^{h} \cap L_{0}^{2}(\Omega)\right)$ and $(\mathbf{u}, \mathbf{v}) \mapsto a(\mathbf{u}, \mathbf{v})$ and $(\mathbf{v}, q) \mapsto b(\mathbf{v}, q)$ stand for the following continuous bilinear forms defined by

$$
\begin{aligned}
& a(\mathbf{u}, \mathbf{v})=\nu \int_{\Omega} \nabla \mathbf{u} \cdot \nabla \mathbf{v} \mathrm{d} x, \quad \mathbf{u}, \mathbf{v} \in\left(H^{1}(\Omega)\right)^{n}, \\
& b(\mathbf{v}, q)=-\int_{\Omega} q \nabla \cdot \mathbf{v} \mathrm{d} x, \quad \mathbf{v} \in\left(H^{1}(\Omega)\right)^{n}, q \in L^{2}(\Omega) .
\end{aligned}
$$

As a rule, usual notation and functional spaces in Partial Differential Equations theory, including Sobolev spaces, are used without further comment (cf., e.g., [18]).

We also assume that the mixed finite element pair $\left(X^{h}, M_{0}^{h}\right)$ satisfies the usual uniform Brezzi stability inf-sup condition, i.e., there exists a positive constant $\beta$, independent of $h$, such that

$$
\sup _{\mathbf{v} \in X^{h}} \frac{b(\mathbf{v}, q)}{\|\mathbf{v}\|_{1, \Omega}} \geq \beta\|q\|_{0, \Omega}, \quad \forall q \in M_{0}^{h} .
$$

\subsection{The domain decomposition method setting}

The mesh $\mathcal{T}^{h}$ is supposed to be compatible with the partition of $\Omega$ into $N$ non-overlapping subdomains $\left\{\Omega_{i}\right\}_{i=1}^{N}$, i.e., $\mathcal{T}^{h}$ induces a local mesh $\mathcal{T}_{i}^{h}$ on each subdomain $\Omega_{i}$, that is, each element of $\mathcal{T}^{h}$ is contained in one of the $\Omega_{i}$. We denote by $\Gamma_{i j}=\bar{\Omega}_{i} \cap \bar{\Omega}_{j}(1 \leq i<j \leq N)$ the interface of two subdomains, whenever it exists (see Fig. 3). For each subdomain, we define $X_{i}^{h}$ as the subspace of $\left(H^{1}\left(\Omega_{i}\right)\right)^{n}$ and $M_{i}^{h}$ as the subspace of $L^{2}\left(\Omega_{i}\right)$, which are generated by the restrictions to $\Omega_{i}$ of functions in respectively $X^{h}$ and $M^{h}$. Note that, from the very definition of $X^{h}$, the elements of $X_{i}^{h}$ have a zero trace on $\partial \Omega_{i} \cap \partial \Omega$.

The description of the DDM considered here, makes use of $X_{B}^{h}$ and $M_{B}^{h}$, two finite element spaces, respectively containing $X^{h}$ and $M^{h}$, whose elements can be discontinuous across the interfaces except at cross points

$$
\begin{gathered}
X_{B}^{h}=\left\{\mathbf{v} \in\left(L^{2}(\Omega)\right)^{n} ;\left.\mathbf{v}\right|_{\Omega_{i}} \in X_{i}^{h}, \mathbf{v} \text { is continuous at cross points }\right\}, \\
M_{B}^{h}=\left\{q \in L^{2}(\Omega) ;\left.q\right|_{\Omega_{i}} \in M_{i}^{h}, q \text { is continuous at cross points }\right\} .
\end{gathered}
$$


In these finite-dimensional spaces, all the subdomains, having in common a cross point, share all the degrees of freedom attached to it. These spaces can be viewed as a "broken" version of the spaces $X^{h}$ and $M^{h}$ where the continuity inter subdomains of the related functions is maintained only at the level of cross points and are respectively endowed in a quite natural way with the norms

$$
\|\mathbf{v}\|_{X_{B}^{h}}=\left\{\sum_{i=1}^{N}\left\|\left.\mathbf{v}\right|_{\Omega_{i}}\right\|_{1, \Omega_{i}}^{2}\right\}^{1 / 2}, \quad\|q\|_{M_{B}^{h}}=\left\{\sum_{i=1}^{N}\left\|\left.q\right|_{\Omega_{i}}\right\|_{0, \Omega_{i}}^{2}\right\}^{1 / 2}=\|q\|_{0, \Omega} .
$$

According to these definitions, every function $\mathbf{v} \in X_{B}^{h}$ can be uniquely decomposed via its nodal values in the following form

$$
\mathbf{v}=\sum_{i=1}^{N} \mathbf{v}_{i}+\mathbf{v}_{c}=\sum_{i=1}^{N}\left(\mathbf{v}_{i 0}+\sum_{j \in \Lambda_{i}} \mathbf{v}_{i j}\right)+\mathbf{v}_{c}
$$

where $\mathbf{v}_{i 0}, \mathbf{v}_{i j}$, and $\mathbf{v}_{c}$ respectively denote the components of $\mathbf{v}$ associated with the nodal values of $\mathbf{v}$ which are respectively in the interior of $\Omega_{i}$, in the interior of the interface $\Gamma_{i j}$ and attached to the cross points. The notation $j \in \Lambda_{i}$ is used to refer to a subdomain $\Omega_{j}$ which shares the interface $\Gamma_{i j}$ with $\Omega_{i}$. Hence $j$ is always different from $i$. It is also worth mentioning that $\mathbf{v}_{i j}$ is zero outside $\Omega_{i}$.

The pressure functions are also decomposed in a similar manner

$$
q=\sum_{i=1}^{N} q_{i}+q_{c}=\sum_{i=1}^{N}\left(q_{i 0}+\sum_{j \in \Lambda_{i}} q_{i j}\right)+q_{c}
$$

Based on this splitting of both the trial and the test functions, we define the following spaces: on the one hand $X_{c}^{h}$ and $M_{c}^{h}$, spanned by the components $\mathbf{v}_{c}^{h} \in X^{h}$ and $q_{c}^{h} \in M^{h}$ respectively and therefore vanishing at all the nodes except those corresponding to a cross point, on the second hand $\dot{X}_{i}^{h}$ and $\dot{M}_{i}^{h}$ the subspaces of functions in $X_{i}^{h}$ and $M_{i}^{h}$ respectively that are vanishing at the cross points.

It is helpful in describing the method to introduce a concise notation for the space of multipliers ensuring the matching of the velocities at the interfaces

$$
W^{h}=\prod_{i=1}^{N} \prod_{j \in \Lambda_{i}} W_{i j}^{h}
$$

where $W_{i j}^{h}$ is spanned by the traces on $\Gamma_{i j}=\Gamma_{j i}$ of the component $\mathbf{v}_{i j}$ of $\mathbf{v}_{i}$ when the latter is running in $X_{i}^{h}$

$$
W_{i j}^{h}=\left\{\Phi_{i j} \in\left(L^{2}\left(\Gamma_{i j}\right)\right)^{n} ; \exists \mathbf{v}_{i} \in X_{i}^{h} \text { such that } \Phi_{i j}=\left.\mathbf{v}_{i j}\right|_{\Gamma_{i j}}\right\}
$$

A similar space is used for dealing with the pressure

$$
Y^{h}=\prod_{i=1}^{N} \prod_{j \in \Lambda_{i}} Y_{i j}^{h}
$$

with

$$
Y_{i j}^{h}=\left\{\psi_{i j} \in L^{2}\left(\Gamma_{i j}\right) ; \exists q_{i} \in M_{i}^{h} \text { such that } \psi_{i j}=\left.q_{i j}\right|_{\Gamma_{i j}}\right\}
$$

Let us stress on the fact that the continuity at the cross points is enforced directly from the definition of the velocity $X_{B}^{h}$ and the pressure $M_{B}^{h}$ spaces. It is important for the sequel to note that any nodal-value corresponding to a cross point of an element in either $W^{h}$ or $Y^{h}$ is zero. 
The interface spaces are endowed with the following norms

$$
\begin{aligned}
\|\cdot\|_{W^{h}} & =\left(\sum_{i=1}^{N} \sum_{j \in \Lambda_{i}} c_{i j}(\cdot, \cdot)\right)^{1 / 2}, \\
\|\cdot\|_{Y^{h}} & =\left(\sum_{i=1}^{N} \sum_{j \in \Lambda_{i}} d_{i j}(\cdot, \cdot)\right)^{1 / 2},
\end{aligned}
$$

which are those induced by the related $L^{2}$-scalar-product on $\Gamma_{i j}$ where $c_{i j}(.,$.$) refers to the vectorial space$ $\left(L^{2}\left(\Gamma_{i j}\right)\right)^{n}$ and $d_{i j}(.,$.$) to the scalar one L^{2}\left(\Gamma_{i j}\right)$.

We can therefore write out the matching conditions for a pair $(\mathbf{u}, p) \in X_{B}^{h} \times M_{B}^{h}$ to belong to $X^{h} \times M^{h}$ in the following form

$$
\left\{\begin{aligned}
c_{i j}\left(\mathbf{u}_{i j}, \Phi_{i j}\right) & =c_{i j}\left(\mathbf{u}_{j i}, \Phi_{i j}\right), & & \forall \Phi_{i j} \in W_{i j}^{h} \\
d_{i j}\left(p_{i j}, \psi_{i j}\right) & =d_{i j}\left(p_{j i}, \psi_{i j}\right), & & \forall \psi_{i j} \in Y_{i j}^{h}
\end{aligned}\right.
$$

which will be more suitable for the description of the DDM.

\subsection{Equivalent formulation of the discrete Stokes equations}

The DDM we consider can be seen as an efficient iterative procedure to solve discrete Stokes problem (1.3). One way of describing this iterative solution is to put it in the form of a fixed-point problem, depending on a parameter $\lambda>0$, consisting in an equivalent writing of problem (1.3) and matching conditions (1.18) on the interfaces. This makes use of the interface unknowns $(\Phi, \psi) \in W^{h} \times Y^{h}$ and is stated in the following theorem.

Theorem 1.1. Let $\lambda>0$ be a given real parameter and $a_{i}(.,),. b_{i}(.,$.$) denote the respective restrictions of the$ bilinear forms $a(.,),. b(.,$.$) to \Omega_{i}$. Then, discrete Stokes problem (1.3) is equivalent to the following system

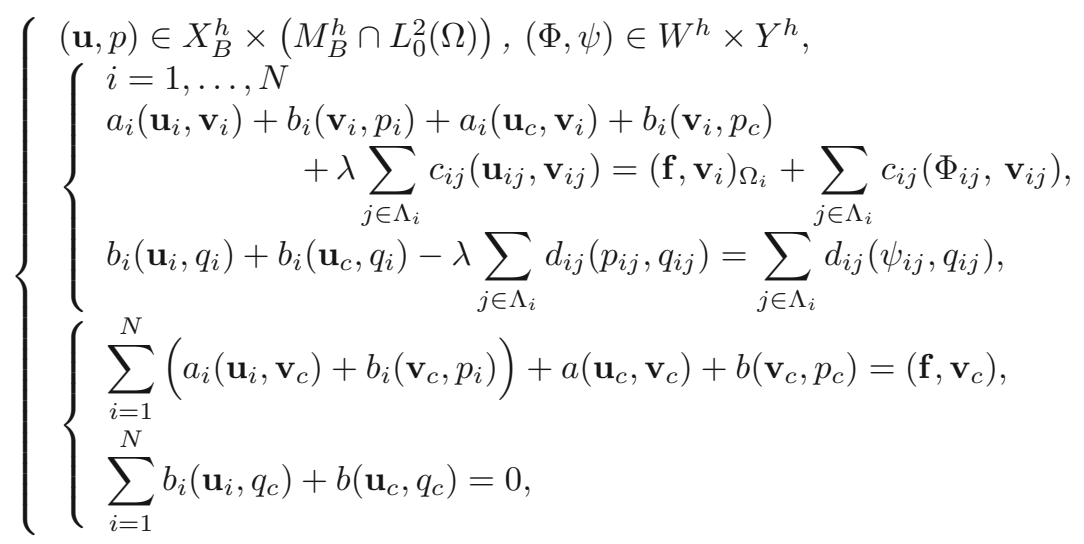

for all $(\mathbf{v}, q) \in X_{B}^{h} \times\left(M_{B}^{h} \cap L_{0}^{2}(\Omega)\right)$. The interface unknowns are subject to the following matching conditions

$$
\Phi_{i j}=-\Phi_{j i}+2 \lambda \mathbf{u}_{j i}, \psi_{i j}=-\psi_{j i}-2 \lambda p_{j i}, \text { for all } i=1, \ldots, N \text { and } j \in \Lambda_{i} .
$$

Proof. The proof is a simple algebraic check obtained by a straightforward adaptation of Proposition 2.1 in [3].

Remark 1.2. It is worth noting that:

- the bilinear forms $d_{i j}(.,$.$) previously considered are irrelevant for a discontinuous-pressure finite element$ method. In this case, there is no need to match the pressures and the broken space $M_{B}^{h}$ is simply the initial space $M^{h}$; 
- first condition in (1.20) actually expresses the matching of both the velocity and the normal stress vector on the interfaces at the discrete level; at the continuous level (see [31]), they take the following form

$$
\nu \frac{\partial \mathbf{u}_{i}}{\partial \mathbf{n}_{i j}}-p_{i} \mathbf{n}_{i j}+\lambda \mathbf{u}_{i}=-\nu \frac{\partial \mathbf{u}_{j}}{\partial \mathbf{n}_{j i}}+p_{j} \mathbf{n}_{j i}+\lambda \mathbf{u}_{j},
$$

where $\lambda>0$ is the parameter considered in Theorem 1.1 and $\mathbf{n}_{i j}$ is the unit normal on $\Gamma_{i j}$ pointing outwards $\Omega_{i}$. The second condition in (1.20) is set to ensure the continuity requirement on the pressure of the finite element scheme. In this way, the whole problem in the velocity and the pressure is dealt with as if it was a usual elliptic coercive system in these two unknowns. We will see how this formal approach can be repeated below to extend the FETI-DP methodology to the discrete problems corresponding to continuous-pressure mixed finite element approximations of the Stokes system.

- Taking $\lambda=0$, that is, considering a Neumann instead of a Fourier-Robin boundary condition at the interfaces gives rise to the two following flaws. Local problems may be non invertible or at least non invertible in a stable way. The equivalence property stated in the above theorem no longer holds true.

\subsection{Main computational cost of an iteration}

The main computational cost for performing an iteration of the DDM algorithm corresponds to the solution of the linear system associated with variational system (1.19) for a given value of the interface unknowns $(\Phi, \psi) \in W^{h} \times Y^{h}$. Denoting by $X_{i}$ the vector of nodal values of $\left(\mathbf{u}_{i}, p_{i}\right)$ and $X_{c}$ the one associated with $\left(\mathbf{u}_{c}, p_{c}\right)$, this system can be put in the following form

$$
\left[\begin{array}{cccc}
A_{11} & & & A_{1 c} \\
& \ddots & & \vdots \\
& & A_{N N} & A_{N c} \\
A_{c 1} & \cdots & A_{c N} & A_{c c}
\end{array}\right]\left[\begin{array}{c}
X_{1} \\
\vdots \\
X_{N} \\
X_{c}
\end{array}\right]=\left[\begin{array}{c}
B_{1} \\
\vdots \\
B_{N} \\
B_{c}
\end{array}\right] .
$$

To efficiently solve this system, we consider its Schur complement associated with $X_{c}$

$$
\begin{gathered}
\left(A_{c c}-\sum_{i=1}^{N} A_{c i} A_{i i}^{-1} A_{i c}\right) X_{c}=B_{c}-\sum_{i=1}^{N} A_{c i} A_{i i}^{-1} B_{i} \\
A_{i i} X_{i}=B_{i}-A_{i c} X_{c}(i=1, \ldots, N) .
\end{gathered}
$$

Each local problem posed on a subdomain is hence solved twice, once for computating the vector $A_{i i}^{-1} B_{i}$ and next to get $X_{i}$ from (1.24). Between these two series of local solutions is inserted an inversion of the linear system (1.23) related to the degrees of freedom supported by the cross points.

Remark 1.3. Actually one can avoid to solve twice the local problems by storing the matrices $A_{i i}^{-1} A_{i c}$. If this way to proceed remains practicable in the $2 \mathrm{D}$ case, it may become redhibitory for $3 \mathrm{D}$ problems.

\section{Stability estimates And CONVERGence Results}

\subsection{Stability of the DDM method}

In this section, we make sure that the mixed DDM algorithm developed above does not break down by proving stability estimates at the level of its various parts where a linear system has to be inverted. Stability is important in several directions. On the one hand, even if it can be proved that the above linear systems are algebraically invertible, the algorithm may not be reliable if their solutions cannot be bounded in terms of the data in suitable norms when $h \rightarrow 0$. On the other hand, the stability result is the crucial tool ensuring the convergence of the iterations corresponding to the fixed-point problem on the interface unknowns. Even if this is not really the most efficient procedure for solving problem (1.20), this convergence is an invaluable 
indicator on the behaviour of the iterative process corresponding to the domain decomposition procedure. The main result of this paper is an estimate of this stability in terms of the $H^{1}$-norm on the subdomains $\Omega_{i}$ and the $L^{2}$-norm on the interfaces $\Gamma_{i j}$ for the space $Y^{h}$. Such a result is hard to improve since the $H^{1}$-norm on the $\Omega_{i}$ is optimal and the $L^{2}$-norm on the interfaces, as far as the pressures are concerned, provides a good control on the invertibility of the involved linear system. However it should be borne in mind that the $L^{2}$-norm on the interfaces cannot be uniformly bounded in $h$ since obviously there is no way to define a trace on the interfaces for a general function in $L^{2}\left(\Omega_{i}\right)$.

We begin by proving a general result ensuring that the local problems can be inverted in a stable way. In what follows $C$ stands for a generic positive constant independent of $h$, which is not the same in all instances.

Theorem 2.1. Under stability assumption (1.6), there exists a constant $C$ independent of $h$ such that every solution to any of the local problems

$$
\left\{\begin{array}{l}
\mathbf{u}_{i} \in \stackrel{\circ}{X}_{i}^{h}, p_{i} \in \stackrel{\circ}{M}_{i}^{h}, \\
a_{i}^{\lambda}\left(\mathbf{u}_{i}, \mathbf{v}_{i}\right)+b_{i}\left(\mathbf{v}_{i}, p_{i}\right)=L_{i} \mathbf{v}_{i}, \quad \forall \mathbf{v}_{i} \in \stackrel{\circ}{X}_{i}^{h} \\
b_{i}\left(\mathbf{u}_{i}, q_{i}\right)-\lambda \sum_{j \in \Lambda_{i}} d_{i j}\left(p_{i j}, q_{i j}\right)=\chi_{i} q_{i}, \quad \forall q_{i} \in \stackrel{\circ}{M}_{i}^{h},
\end{array}\right.
$$

with $L_{i}$ and $\chi_{i}$ two linear forms on respectively $X_{i}^{h}$ and $M_{i}^{h}$ and

$$
a_{i}^{\lambda}\left(\mathbf{u}_{i}, \mathbf{v}_{i}\right)=a_{i}\left(\mathbf{u}_{i}, \mathbf{v}_{i}\right)+\lambda \sum_{j \in \Lambda_{i}} c_{i j}\left(\mathbf{u}_{i j}, \mathbf{v}_{i j}\right),
$$

satisfies

$$
\left\|\mathbf{u}_{i}\right\|_{1, \Omega_{i}}+\left\|p_{i}\right\|_{0, \bar{\Omega}_{i}} \leq C\left(\sup _{\left\|\mathbf{v}_{i}\right\|_{1, \Omega_{i}} \leq 1}\left|L_{i} \mathbf{v}_{i}\right|+\sup _{\left\|q_{i}\right\|_{0, \bar{\Omega}_{i}} \leq 1}\left|\chi_{i} q_{i}\right|\right),
$$

where $\left\|p_{i}\right\|_{0, \bar{\Omega}_{i}}$ is the graph norm on the space $M_{i}^{h}$ involving the usual $L^{2}$-norm on $\Omega_{i}$ and the $L^{2}$-norm on the interfaces

$$
\left\|p_{i}\right\|_{0, \bar{\Omega}_{i}}=\sqrt{\left\|p_{i}\right\|_{0, \Omega_{i}}^{2}+\sum_{j \in \Lambda_{i}} d_{i j}\left(p_{i j}, p_{i j}\right)} .
$$

Proof. It must be first noted that all the involved bilinear forms are uniformly bounded in terms of the $H^{1}$ norm on $\Omega_{i}$ and the graph norm introduced in the statement of the theorem. However, problem (2.1) does not completely fall within the framework of the mixed finite element formulation (cf., e.g., [8]) since the second stability inf-sup condition of Brezzi: $\exists \beta>0$ independent of $h$ such that

$$
\sup _{\left\|\mathbf{v}_{i}\right\|_{1, \Omega_{i}} \leq 1} b\left(\mathbf{v}_{i}, q_{i}\right) \geq \beta\left\|q_{i}\right\|_{0, \Omega_{i}}, \quad \forall q_{i} \in \stackrel{\circ}{M}_{i}^{h}
$$

holds in a weaker norm only. The way of obtaining this estimate is recalled in the rest of this proof. We have therefore to carefully check the various arguments enabling us to get bound (2.3). At first, note that since each part of the interface strictly contains an open set $\omega_{i j}$, at least for $h$ small enough, the usual Poincaré inequality ensures that the bilinear form $a_{i}^{\lambda}(\cdot, \cdot)$ is uniformly coercive on $H^{1}\left(\Omega_{i}\right)$

$$
a_{i}^{\lambda}\left(\mathbf{v}_{i}, \mathbf{v}_{i}\right) \geq \nu\left|\mathbf{v}_{i}\right|_{1, \Omega_{i}}^{2}+\lambda \sum_{j \in \Lambda_{i}}\left|\mathbf{v}_{i}\right|_{0, \omega_{i j}}^{2} \geq \alpha\left\|\mathbf{v}_{i}\right\|_{1, \Omega_{i}}^{2},
$$

where $\alpha>0$ is a constant independent of $h$. By " $\omega_{i j}$ is strictly contained in $\Gamma_{i j}$ ", we mean that its closure $\overline{\omega_{i j}}$ is contained in the interior of $\Gamma_{i j}$. Let us say some words now on the proof of (2.5). It can be obtained by a straightforward adaptation of the one given in [34] for the case of a domain with mixed Dirichlet-Neumann 
boundary condition. The main step is to pick one subdomain $\Omega_{j}\left(j \in \Lambda_{i}\right)$ sharing an interface with $\Omega_{i}$ and to observe that any function $\mathbf{v}_{i} \in X_{i}^{h}$ having a trace equal to zero on $\partial \Omega_{i}$ except on $\Gamma_{i j}$ actually belongs to $X_{i}^{h}$. The proof is then obtained in the same way as in [34] (Prop. 5.3.2). We now focus on estimate (2.3). An obvious combination of the equations in (2.1) gives

$$
a_{i}^{\lambda}\left(\mathbf{u}_{i}, \mathbf{u}_{i}\right)+\lambda \sum_{j \in \Lambda_{i}} d_{i j}\left(p_{i j}, p_{i j}\right)=L_{i} \mathbf{u}_{i}-\chi_{i} p_{i} .
$$

We then readily get the following bound

$$
\left\|\mathbf{u}_{i}\right\|_{1, \Omega_{i}}^{2}+\sum_{j \in \Lambda_{i}} d_{i j}\left(p_{i j}, p_{i j}\right) \leq C\left(\sup _{\left\|\mathbf{v}_{i}\right\|_{1, \Omega_{i}} \leq 1}\left|L_{i} \mathbf{v}_{i}\right|+\sup _{\left\|q_{i}\right\|_{0, \bar{\Omega}_{i}} \leq 1}\left|\chi_{i} q_{i}\right|\right)\left(\left\|\mathbf{u}_{i}\right\|_{1, \Omega_{i}}^{2}+\left\|p_{i}\right\|_{0, \bar{\Omega}_{i}}^{2}\right)^{1 / 2} .
$$

Clearly we have not enough control, at this level, on the graph norm of $p_{i}$. We then use the above inf-sup condition to get

$$
\beta\left\|p_{i}\right\|_{0, \Omega_{i}} \leq C_{a_{i}^{\lambda}}\left\|\mathbf{u}_{i}\right\|_{1, \Omega_{i}}+\sup _{\left\|\mathbf{v}_{i}\right\|_{1, \Omega_{i}} \leq 1}\left|L_{i} \mathbf{v}_{i}\right|
$$

where $C_{a_{i}^{\lambda}}$ is the continuity constant of $a_{i}^{\lambda}(\cdot, \cdot)$ on $H^{1}\left(\Omega_{i}\right) \times H^{1}\left(\Omega_{i}\right)$. Here we have used the fact that $\left.\mathbf{u}_{i}\right|_{\Gamma_{i j}}=$ $\left.\mathbf{u}_{i j}\right|_{\Gamma_{i j}}$ since $\mathbf{u}_{i}$ is assumed to be in $\AA_{i}^{h}$. Considering the above two inequalities, we first get

$$
\left\|p_{i}\right\|_{0, \Omega_{i}} \leq C\left(\sup _{\left\|\mathbf{v}_{i}\right\|_{1, \Omega_{i}} \leq 1}\left|L_{i} \mathbf{v}_{i}\right|+\sup _{\left\|q_{i}\right\|_{0, \Omega_{i}} \leq 1}\left|\chi_{i} q_{i}\right|\right)
$$

and then come to

$$
\left\|\mathbf{u}_{i}\right\|_{1, \Omega_{i}}^{2}+\left\|p_{i}\right\|_{0, \bar{\Omega}_{i}}^{2} \leq C\left(\begin{array}{c}
\left(\sup _{\left\|\mathbf{v}_{i}\right\|_{1, \Omega_{i}} \leq 1}\left|L_{i} \mathbf{v}_{i}\right|+\sup _{\left\|q_{i}\right\|_{0, \bar{\Omega}_{i}} \leq 1}\left|\chi_{i} q_{i}\right|\right)^{2} \\
+\left(\sup _{\left\|\mathbf{v}_{i}\right\|_{1, \Omega_{i}} \leq 1}\left|L_{i} \mathbf{v}_{i}\right|+\sup _{\left\|q_{i}\right\|_{0, \bar{\Omega}_{i}} \leq 1}\left|\chi_{i} q_{i}\right|\right)\left(\left\|\mathbf{u}_{i}\right\|_{1, \Omega_{i}}^{2}+\left\|p_{i}\right\|_{0, \bar{\Omega}_{i}}^{2}\right)^{1 / 2}
\end{array}\right)
$$

The proof is then completed by an elementary argument on the sign of a quadratic polynomial.

Remark 2.2. Recall that $q \in \stackrel{\circ}{M}_{i}^{h}$ means that $q \in M_{i}^{h}$ and that its nodal values associated to a cross point are zero. This property plays no role when establishing the above local inf-sup condition (2.5) and is therefore valid for $q \in M_{i}^{h}$. This will be of importance below for the stability of the DDM algorithm.

As this was mentioned above for the implementation of the mixed DDM considered here, a linear system related to the degrees of freedom attached to the cross points is inverted between two solutions of the local problems. Thus, we must prove that this system can be solved in a stable way. Since we have already shown that the solution of the local problems is stable, it is enough to prove that the global system, involving local degrees of freedom as well as those supported by the cross points, carries similar stability properties.

To achieve this goal, we need first to make more precise the definition of the augmentation of the local equations. This is done by noting that the components $\mathbf{v}_{i j}$ of $\mathbf{v}_{i} \in X_{i}^{h}$ in (1.10) can be obtained by means of algebraic projectors defined as follows: $\pi_{i j} \Phi$ has the same degrees of freedom as $\Phi$ except those attached to a cross point which are set to zero, $\Phi$ being here equal to the trace $\left.\mathbf{v}_{i}\right|_{\Gamma_{i j}}$ of $\mathbf{v}_{i} \in X_{i}^{h}$. 
The component $q_{i j}\left(i=1, \ldots, N, j \in \Lambda_{i}\right)$ of $q \in M_{B}^{h}$ in (1.11) can be obtained from algebraic projectors $\sigma_{i j}$ defined in the same way. We are thus in a position to give a suitable statement of the global problem

$$
\left\{\begin{array}{l}
\mathbf{u} \in X_{B}^{h}, p \in M_{B}^{h} \cap L_{0}^{2}(\Omega), \\
\sum_{i=1}^{N}\left(a_{i}(\mathbf{u}, \mathbf{u})+b_{i}(\mathbf{v}, p)\right)+\lambda \sum_{i=1}^{N} \sum_{j \in \Lambda_{i}} c_{i j}\left(\pi_{i j} \mathbf{u}, \pi_{i j} \mathbf{v}\right)=L \mathbf{v}, \forall \mathbf{v} \in X_{B}^{h}, \\
\sum_{i=1}^{N} b_{i}(\mathbf{u}, q)-\lambda \sum_{i=1}^{N} \sum_{j \in \Lambda_{i}} d_{i j}\left(\sigma_{i j} p, \sigma_{i j} q\right)=\chi q, \quad \forall q \in M_{B}^{h} \cap L_{0}^{2}(\Omega),
\end{array}\right.
$$

where $L$ and $\chi$ are linear forms on respectively $X_{B}^{h}$ and $M_{B}^{h}$ arbitrarily chosen. In (2.12), to shorten the notation, the restriction to $\Omega_{i}$, like for instance $\left.\mathbf{u}\right|_{\Omega_{i}}$ for $\mathbf{u} \in X_{B}^{h}$, is left implicit.

As usual, the stability of problem (2.12) is obtained in the framework of mixed formulations. The two stability Brezzi conditions, the coercivity of the bilinear form on $X_{B}^{h} \times X_{B}^{h}$ and the inf-sup condition on $X_{B}^{h} \times$ $\left(M_{B}^{h} \cap L_{0}^{2}(\Omega)\right)$ will be obtained as a simple consequence of the corresponding local properties established in Theorem 2.1. Surprisingly enough, it is now a uniform bound for the continuity of the bilinear forms $a_{i}^{\lambda}(\cdot, \cdot)$ which is less straightforward to establish, because of the algebraic operators that are used in its definition. We prove such a bound under a technical hypothesis. For the particular case of the Taylor-Hood elements, this hypothesis is the consequence of a regularity assumption on the meshes as usual in the finite element method (cf., e.g., $[7,10])$.

Lemma 2.3. Assuming that the finite element space for approximating the velocities fulfills the following conditions:

- it induces a finite element space on the boundary;

- each triple $\left\{T, P_{T}, \Sigma_{T}\right\}$ associated to any geometrical element $T$ on an interface in the terminology of the analysis of the finite element method (cf., e.g., [10]) can be obtained from a reference finite element $\{\widehat{T}, \widehat{P}, \widehat{\Sigma}\}$ from a smooth bijection $F_{T}: \widehat{T} \rightarrow T$

- its Jacobian $F_{T}^{\prime}$ satisfies the following bound

$$
\max _{\widehat{x} \in \widehat{T}}\left|\operatorname{det} F_{T}^{\prime} \widehat{x}\right| / \min _{\widehat{x} \in \widehat{T}}\left|\operatorname{det} F_{T}^{\prime} \widehat{x}\right| \leq C,
$$

with a constant independent of $h$.

Then, there exists a constant independent of $h$ such that

$$
\left|\sum_{i=1}^{N}\left(a_{i}(\mathbf{u}, \mathbf{v})+\lambda \sum_{j \in \Lambda_{i}} c_{i j}\left(\pi_{i j} \mathbf{u}, \pi_{i j} \mathbf{v}\right)\right)\right| \leq C\|\mathbf{u}\|_{X_{B}^{h}}\|\mathbf{v}\|_{X_{B}^{h}}
$$

for all $\mathbf{u}$ and $\mathbf{v}$ in $X_{B}^{h}$.

Proof. The proof will be a simple consequence of the following bound: there exists a constant independent of $h$ such that

$$
\int_{T}\left|\pi_{i j} \mathbf{u}\right|^{2} \mathrm{~d} x \leq C \int_{T}|\mathbf{u}|^{2} \mathrm{~d} x
$$

for all geometrical elements $T$ contained in an interface $\Gamma_{i j}$. If $T$ contains no cross point, $\pi_{i j}$ is simply the identity operator. Otherwise, the hypothesis on the existence of a reference element makes it possible to transport the definition $\pi_{i j}$ on another algebraic projector $\widehat{\pi}$ defined on $\widehat{T}$ such that the transformation defined by

$$
\widehat{\mathbf{u}}(\widehat{x})=\mathbf{u}\left(F_{T} \widehat{x}\right),
$$


makes it possible to write

$$
\widehat{\pi_{i j} \mathbf{u}}=\widehat{\pi} \widehat{\mathbf{u}}
$$

We thus have

$$
\int_{T}\left|\pi_{i j} \mathbf{u}\right|^{2} \mathrm{~d} x \leq \max _{\widehat{x} \in \widehat{T}}\left|\operatorname{det} F_{T}^{\prime} \widehat{x}\right| \int_{\widehat{T}}\left|\widehat{\pi_{i j} \mathbf{u}}\right|^{2} \mathrm{~d} \widehat{x}=\max _{\widehat{x} \in \widehat{T}}\left|\operatorname{det} F_{T}^{\prime} \widehat{x}\right| \int_{\widehat{T}}|\widehat{\pi} \widehat{\mathbf{u}}|^{2} \mathrm{~d} \widehat{x} .
$$

But since $\widehat{\pi}$ is a linear mapping from the finite dimensional space $\widehat{P}$ into itself, we first get

$$
\int_{\widehat{T}}|\widehat{\pi} \widehat{\mathbf{u}}|^{2} \mathrm{~d} \widehat{x} \leq C \int_{\widehat{T}}|\widehat{\mathbf{u}}|^{2} \mathrm{~d} \widehat{x}
$$

with a constant depending only on the reference element and $\widehat{P}$. This directly yields

$$
\int_{T}\left|\pi_{i j} \mathbf{u}\right|^{2} \mathrm{~d} x \leq C \max _{\widehat{x} \in \widehat{T}}\left|\operatorname{det} F_{T}^{\prime} \widehat{x}\right| \int_{\widehat{T}}|\widehat{\mathbf{u}}|^{2} \mathrm{~d} \widehat{x} .
$$

In the same way, coming back to an integral on $T$, we obtain

$$
\int_{T}\left|\pi_{i j} \mathbf{u}\right|^{2} \mathrm{~d} x \leq C \frac{\max _{\widehat{x} \in \widehat{T}}\left|\operatorname{det} F_{T}^{\prime} \widehat{x}\right|}{\min _{\widehat{x} \in \widehat{T}}\left|\operatorname{det} F_{T}^{\prime} \widehat{x}\right|} \int_{T}|\mathbf{u}|^{2} \mathrm{~d} x .
$$

The proof is then a simple consequence of the properties assumed on the velocity finite element.

Lemma 2.4. The bilinear forms involved in system (2.12) satisfy the following Brezzi stability conditions

$$
\begin{gathered}
\sum_{i=1}^{N}\left(a_{i}(\mathbf{u}, \mathbf{v})+\lambda \sum_{j \in \Lambda_{i}} \int_{\Gamma_{i j}}\left|\pi_{i j} \mathbf{u}\right|^{2} \mathrm{~d} x\right) \geq \alpha\|\mathbf{u}\|_{X_{B}^{h}}^{2} \quad \forall \mathbf{u} \in X_{B}^{h}, \\
\sup _{\|\mathbf{v}\|_{X_{B}^{h}} \leq 1} \sum_{i=1}^{N} b_{i}(\mathbf{v}, q) \geq \beta\|q\|_{0, \Omega} \quad \forall q \in M_{B}^{h},
\end{gathered}
$$

where $\alpha$ and $\beta$ are positive constants independent of $h$.

Proof. Bound (2.22) is obtained by a straightforward extension of the proof given in Theorem 2.1. To establish (2.23), we fix $q$ in $M_{B}^{h}$ and use the observation made in Remark 2.2 to assert that there exists $\beta_{i}$ independent of $h$ and $\mathbf{v}_{i} \in \stackrel{\circ}{X}_{i}^{h}$ which vanishes on $\partial \Omega_{i}$ but not on one of the interfaces $\Gamma_{i j}$ such that $\left\|\mathbf{v}_{i}\right\|_{1, \Omega_{i}}=1$ and

$$
b_{i}\left(\mathbf{v}_{i}, q\right) \geq \beta_{i}\|q\|_{0, \Omega_{i}}
$$

We can assume that $q \neq 0$, otherwise the inequality is satisfied by taking an arbitrary constant $\beta>0$. Setting $\beta=\min _{1 \leq i \leq N} \beta_{i}$, we readily get

$$
\sum_{i=1}^{N} \frac{\|q\|_{0, \Omega_{i}}}{\|q\|_{0, \Omega}} b_{i}\left(\mathbf{v}_{i}, q\right) \geq \frac{\beta}{\|q\|_{0, \Omega}} \sum_{i=1}^{N}\|q\|_{0, \Omega_{i}}^{2}=\beta\|q\|_{0, \Omega}
$$

Defining $\mathbf{v}$ on $\Omega$ by $\left.\mathbf{v}\right|_{\Omega_{i}}=\left(\|q\|_{0, \Omega_{i}} /\|q\|_{0, \Omega}\right) \mathbf{v}_{i}$, we can assert that $\mathbf{v} \in X_{B}^{h}$ since it is equal to zero at the cross points. The end of the proof is then immediate.

We can now prove the main stability property of the mixed DDM developed here. In particular, this ensures that the algorithm cannot break down and that the related iterative procedure converges. 
Theorem 2.5. There exists a constant $C$ independent of $h$ such that for any couple of linear forms $L$ and $\chi$ respectively on $X_{B}^{h}$ and $M_{B}^{h}$ problem (2.12) has one and only one solution satisfying

$$
\|\mathbf{u}\|_{X_{B}^{h}}+\sqrt{\|p\|_{0, \Omega}^{2}+\sum_{i=1}^{N} \sum_{j \in \Lambda_{i}} d_{i j}\left(\sigma_{i j} p, \sigma_{i j} p\right)} \leq C\left(\sup _{\|\mathbf{v}\|_{X_{B}^{h}} \leq 1}|L \mathbf{v}|+\sqrt{\sqrt{\|q\|_{0, \Omega}^{2}+\sum_{i=1}^{N} \sum_{j \in \Lambda_{i}} d_{i j}\left(\sigma_{i j} q, \sigma_{i j} q\right)} \leq 1}|\chi q|\right) .
$$

Proof. The proof is obtained along the same path as that of Theorem 2.1 from above estimates (2.14), (2.22) and (2.23).

Remark 2.6. Defining the linear forms $L$ and $\chi$ by

$$
L \mathbf{v}=\sum_{i=1}^{N}\left((\mathbf{f}, \mathbf{v})_{\Omega_{i}}+\sum_{j \in \Lambda_{i}} c_{i j}\left(\Phi_{i j}, \pi_{i j} \mathbf{v}\right)\right), \chi q=\sum_{i=1}^{N} \sum_{j \in \Lambda_{i}} d_{i j}\left(\psi_{i j}, \sigma_{i j} q\right)
$$

and noting that

$$
\begin{aligned}
& |L \mathbf{v}| \leq C\left(\|\mathbf{f}\|_{0, \Omega}^{2}+\|\Phi\|_{W^{h}}^{2}\right)^{1 / 2}\|\mathbf{v}\|_{X_{B}^{h}}, \forall \mathbf{v} \in X_{B}^{h}, \\
& |\chi q| \leq\|\psi\|_{Y^{h}}\left(\sum_{i=1}^{N} \sum_{j \in \Lambda_{i}} d_{i j}\left(\sigma_{i j} q, \sigma_{i j} q\right)\right)^{1 / 2}, \forall q \in M_{B}^{h},
\end{aligned}
$$

we obtain that the above theorem actually establishes that problem (1.19) can be solved in a stable way.

Corollary 2.7. Any iteration of the mixed DDM algorithm can be done in a stable way by solving the local problems and the one related to the cross points in the following meaning: the stability estimate (2.26) is valid in the following sharper manner

$$
\begin{aligned}
& \left\|\mathbf{u}_{i 0}+\sum_{j \in \Lambda_{i}} \mathbf{u}_{i j}\right\|_{X_{i}^{h}}+\left\|p_{i 0}+\sum_{j \in \Lambda_{i}} p_{i j}\right\|_{0, \bar{\Omega}_{i}} \leq C\left(\sup _{\|\mathbf{v}\|_{X_{B}^{h}} \leq 1}|L \mathbf{v}|+\sup _{\sqrt{\|q\|_{0, \Omega}^{2}+\sum_{i=1}^{N} \sum_{j \in \Lambda_{i}} d_{i j}\left(\sigma_{i j} q, \sigma_{i j} q\right)} \leq 1}|\chi q|\right) \\
& (i=1, \ldots, N) \\
& \left\|\mathbf{u}_{c}\right\|_{X_{B}^{h}}+\sqrt{\left\|p_{c}\right\|_{0, \Omega}^{2}+\sum_{i=1}^{N} \sum_{j \in \Lambda_{i}} d_{i j}\left(\sigma_{i j} p_{c}, \sigma_{i j} p_{c}\right)} \leq C\left(\sup _{\|\mathbf{v}\|_{X_{B}^{h}} \leq 1}|L \mathbf{v}|+\frac{\sup _{\sqrt{\|q\|_{0, \Omega}^{2}+\sum_{i=1}^{N} \sum_{j \in \Lambda_{i}} d_{i j}\left(\sigma_{i j} q, \sigma_{i j} q\right)} \leq 1}|\chi q|}{)}\right)
\end{aligned}
$$

where $\mathbf{u}=\sum_{i=1}^{N}\left(\mathbf{u}_{i 0}+\sum_{j \in \Lambda_{i}} \mathbf{u}_{i j}\right)+\mathbf{u}_{c}$ and $p=\sum_{i=1}^{N}\left(p_{i 0}+\sum_{j \in \Lambda_{i}} p_{i j}\right)+p_{c}$ are the decomposition of respectively $\mathbf{u}$ and $p$ already respectively defined in (1.10) and (1.11).

Proof. By taking $(\mathbf{v}, q)=\left(\mathbf{v}_{i 0}+\sum_{j \in \Lambda_{i}} \mathbf{v}_{i j}, q_{i 0}+\sum_{j \in \Lambda_{i}} q_{i j}\right)$ as testing function, we directly get from the local stability Theorem 2.1

$$
\left\|\mathbf{u}_{i 0}+\sum_{j \in \Lambda_{i}} \mathbf{u}_{i j}\right\|_{X_{i}^{h}}+\left\|p_{i 0}+\sum_{j \in \Lambda_{i}} p_{i j}\right\|_{0, \bar{\Omega}_{i}} \leq C\left(\begin{array}{c}
\sup _{\|\mathbf{v}\|_{X_{B}^{h}} \leq 1}|L \mathbf{v}|+\frac{\sup }{\sqrt{\|q\|_{0, \Omega}^{2}+\sum_{i=1}^{N} \sum_{j \in \Lambda_{i}} d_{i j}\left(\sigma_{i j} q, \sigma_{i j} q\right)} \leq 1} \\
+\left\|\mathbf{u}_{c}\right\|_{X_{B}^{h}}+\sqrt{\left\|p_{c}\right\|_{0, \Omega}^{2}+\sum_{i=1}^{N} \sum_{j \in \Lambda_{i}} d_{i j}\left(\sigma_{i j} p_{c}, \sigma_{i j} p_{c}\right)}
\end{array}\right) .
$$

The proof can then be easily completed by using the triangle inequality and estimate (2.26). 


\subsection{Convergence of the mixed DDM algorithm}

Let us recall that the actual DDM consists in reducing the linear system obtained from equations (1.19) and (1.20) to an implicit one but related to the interface unknowns only

$$
(\Phi, \psi)=S((\Phi, \psi), \mathbf{f}),
$$

where $(\Theta, \zeta)=S((\Phi, \psi), \mathbf{f})$ is obtained by solving (1.19) in $\mathbf{u}$ and $p, \Phi, \psi$ and $\mathbf{f}$ being hence given, and, next, forming the new system of interface data $(\Theta, \zeta)$ by

$$
\Theta_{i j}=-\Phi_{j i}+2 \lambda \mathbf{u}_{j i}, \zeta_{i j}=-\psi_{j i}-2 \lambda p_{j i}
$$

The linear system associated to (2.32) is best solved by a Krylov method. This procedure requires to know the right-hand side and a way to perform the associated matrix-vector product corresponding to the actual linear system. Indeed the linear problem (2.32) can be written in the following form

$$
(\Phi, \psi)-S((\Phi, \psi), 0)=S((0,0), \mathbf{f})
$$

which more clearly indicates that the right-hand side of the corresponding linear system can be obtained by evaluating $S((0,0), \mathbf{f})$, that is, by performing the first of the successive approximations starting from zero as initial guess and that the associated matrix-vector product as the difference of two successive iterates corresponding to the system of driven forces $\mathbf{f}=0$, that is, $(\Phi, \psi)-S((\Phi, \psi), 0)$. Of course once (2.32) becomes (approximatively) solved, one can recover the (approximate) solution of the Stokes problem.

However, as a fixed point problem, system $(2.32)$ can be solved by the related relaxation scheme depending on a parameter $0<\theta<1$ :

- $\left(\Phi^{(0)}, \psi^{(0)}\right)$ being fixed as an initial guess;

- for $k=0,1,2, \ldots$ until convergence do

$$
\left(\Phi^{(k+1)}, \psi^{(k+1)}\right)=(1-\theta)\left(\Phi^{(k)}, \psi^{(k)}\right)+\theta S\left(\left(\Phi^{(k)}, \psi^{(k)}\right), \mathbf{f}\right) .
$$

As just mentioned above, even if this procedure is not the most efficient for solving (2.32), its convergence properties give an important indication on the convergence of the more efficient ones generally based on a Krylov method.

The key tool for establishing this convergence is provided by a technique of proof introduced by Collino et al. [13] and next stated in a general framework in [3]. We can thus state and prove the main convergence theorem for the DDM considered here.

Theorem 2.8. Let the sequence $\left(\mathbf{u}^{(k)}, p^{(k)}\right)$ be defined recursively during the above relaxation algorithm. Then $\left(\mathbf{u}^{(k)}, p^{(k)}\right)$ converges towards the solution $(\mathbf{u}, p)$ to discrete Stokes problem (1.3) with the following error bound

$$
\left(\left\|\mathbf{u}^{(k)}-\mathbf{u}\right\|_{X_{B}^{h}}^{2}+\left\|p^{(k)}-p\right\|_{0, \Omega}^{2}\right)^{1 / 2} \leq C \kappa_{h}^{k}\left(\begin{array}{l}
\left\|\mathbf{u}^{(0)}-\mathbf{u}\right\|_{X_{B}^{h}}^{2}+\left\|p^{(0)}-p\right\|_{0, \Omega}^{2} \\
+\sum_{i=1}^{N} \sum_{j \in \Lambda_{i}} d_{i j}\left(\sigma_{i j}\left(p^{(0)}-p\right), \sigma_{i j}\left(p^{(0)}-p\right)\right)
\end{array}\right)^{1 / 2}
$$

where $\kappa_{h}$ is a constant $<1$ possibly depending on $h$.

Proof. According to Lemma 3.2 in [3] the mapping

$$
(\Phi, \psi) \mapsto(1-\theta)(\Phi, \psi)+\theta S((\Phi, \psi), \mathbf{f})
$$

is a strict contraction, if $S$ does not augment the norm in the following meaning

$$
\|S((\Phi, \psi), 0)\|_{W^{h} \times Y^{h}} \leq\|(\Phi, \psi)\|_{W^{h} \times Y^{h}},
$$


and satisfies the left-bound

$$
\exists \gamma>0, \quad\|(\Phi, \psi)-S((\Phi, \psi), 0)\|_{W^{h} \times Y^{h}} \geq \gamma\|(\Phi, \psi)\|_{W^{h} \times Y^{h}},
$$

for all $(\Phi, \psi) \in W^{h} \times Y^{h}$. The contraction constant of this mapping is then given by $\kappa_{h}=\sqrt{1-\theta(1-\theta) \gamma^{2}}$.

Since $W^{h}, Y^{h}$ are both finite dimensional spaces, condition (2.38) is equivalent to the invertibility of the mapping $(\Phi, \psi) \rightarrow(\Phi, \psi)-S((\Phi, \psi), 0)$ and thus to the following condition

$$
S((\Phi, \psi), 0)=(\Phi, \psi) \text { if and only if }(\Phi, \psi)=(0,0) .
$$

It is worth noting that the above constant $\gamma$ depends on the mesh size $h$ since its existence will be obtained through an algebraic argument only. Hence let then $(\mathbf{u}, p)$ and $(\Phi, \psi)$ be a solution to (1.19) and (1.20) with $\mathbf{f}=0$. The pair $(\mathbf{u}, p)$ is thus a solution to discrete Stokes system (1.3) with zero as second member. It is therefore equal to 0 . We thus obtain

$$
\sum_{i=1}^{N} \sum_{j \in \Lambda_{i}} c_{i j}\left(\Phi_{i j}, \pi_{i j} \mathbf{v}\right)=0, \forall \mathbf{v} \in X_{B}^{h}
$$

and

$$
\sum_{i=1}^{N} \sum_{j \in \Lambda_{i}} d_{i j}\left(\psi_{i j}, \sigma_{i j} q\right)=0, \forall q \in M_{B}^{h} \cap L_{0}^{2}(\Omega) .
$$

By choosing $q \in M_{B}^{h}$ such that $\left.q\right|_{\Gamma_{i j}}=\psi_{i j}$ and possibly suitably fixing a value for $q$ at a node internal to some $\Omega_{i}$, this in particular implies that $d_{i j}\left(\psi_{i j}, \psi_{i j}\right)=0$ and thus $\psi=0$. The fact that $\Phi=0$ is easier since no condition is imposed on $\mathbf{v}$. To establish property $(2.37)$, we first expand $\|S((\Phi, \psi), 0)\|_{W^{h} \times Y^{h}}^{2}$ as

$$
\begin{aligned}
\|S((\Phi, \psi), 0)\|_{W^{h} \times Y^{h}}^{2}= & \sum_{i=1}^{N} \sum_{j \in \Lambda_{i}}\left(\left\|-\Phi_{i j}+2 \lambda \mathbf{u}_{i j}\right\|_{0, \Gamma_{i j}}^{2}+\left\|-\psi_{i j}-2 \lambda p_{i j}\right\|_{0, \Gamma_{i j}}^{2}\right) \\
= & \sum_{i=1}^{N} \sum_{j \in \Lambda_{i}}\left(\left(\left\|\Phi_{i j}\right\|_{0, \Gamma_{i j}}^{2}-4 \lambda c_{i j}\left(\Phi_{i j}, \mathbf{u}_{i j}\right)+4 \lambda^{2}\left\|\mathbf{u}_{i j}\right\|_{0, \Gamma_{i j}}^{2}\right)\right. \\
& \left.+\left(\left\|\psi_{i j}\right\|_{0, \Gamma_{i j}}^{2}+4 \lambda d_{i j}\left(\psi_{i j}, p_{i j}\right)+4 \lambda^{2}\left\|p_{i j}\right\|_{0, \Gamma_{i j}}^{2}\right)\right) .
\end{aligned}
$$

Since $(\mathbf{u}, p)$ is a solution to problem (2.12) with $L \mathbf{v}=\sum_{i=1}^{N} \sum_{j \in \Lambda_{i}} c_{i j}\left(\Phi_{i j}, \pi_{i j} \mathbf{v}\right)$ and $\chi q=\sum_{i=1}^{N} \sum_{j \in \Lambda_{i}} d_{i j}\left(\psi_{i j}, \sigma_{i j} q\right)$, we therefore obtain

$$
\|S((\Phi, \psi), 0)\|_{W^{h} \times Y^{h}}^{2}=\|(\Phi, \psi)\|_{W^{h} \times Y^{h}}^{2}-4 \lambda \sum_{i=1}^{N} a_{i}(\mathbf{u}, \mathbf{u}) .
$$

Error bound (2.35) directly follows from stability Theorem 2.5.

Remark 2.9. The contraction constant $0<\kappa_{h}=\sqrt{1-\theta(1-\theta) \gamma^{2}}<1$ depends on the mesh size $h$ through the parameter $\gamma$. Its best value thus corresponds to $\theta=1 / 2$. As this is reported in [13] for the Helmholtz equation, it is necessary to replace $\lambda$ by a suitable nonlocal operator so that $\gamma$ and hence the contraction constant become independent of $h$. The mixed DDM algorithm may then become much more difficult to implement without bringing a decisive advantage in the efficiency as this was observed in [5]. For the test-case considered below, we shall see however how to adapt some algebraic techniques used in the context of usual coercive problems to speed-up the iterative process. A thorough study of such a manner to proceed will be presented in a forthcoming work. 
TABLE 1. Relative errors corresponding to various meshes and a direct solution of the discrete system.

\begin{tabular}{|c|c|c|c|c|c|}
\hline$h$ & Triangles & d.o.f. u & d.o.f. $p$ & $\operatorname{Err}(u)=10^{-5} *$ & $\operatorname{Err}(p)=10^{-3} *$ \\
\hline $1 / 10$ & 292 & 1266 & 171 & 269 & 165 \\
$1 / 20$ & 1406 & 5794 & 746 & 57 & 29 \\
$1 / 30$ & 2980 & 12162 & 1551 & 25 & 12 \\
$1 / 40$ & 5400 & 21931 & 2785 & 14 & 7 \\
$1 / 50$ & 8412 & 34058 & 4309 & 9 & 5 \\
$1 / 60$ & 55070 & 49706 & 6274 & 6 & 2 \\
\hline
\end{tabular}

\section{NUMERICAL EXPERIMENTS}

\subsection{The considered test-case}

The numerical experiments were conducted using the test-case devised by Bercovier and Engelman [4]. In this example, the problem is posed on the unit square $\Omega=] 0,1[\times] 0,1[$. The driven forces are calculated from the following polynomial expressions for the velocity $\mathbf{u}(x, y)=[w(x, y),-w(y, x)]^{t}$, with $w(x, y)=$ $x^{2}(x-1)^{2} y(y-1)(2 y-1)$, the pressure $p(x, y)=(x-1 / 2)(y-1 / 2)$ and $\nu=1$.

The mixed finite element method used is the popular Taylor-Hood element pair on a triangular mesh with quadratic velocities (see for instance [8]). The zero mean value condition (1.2) is taken into account by means of a Lagrange multiplier. It is generally difficult to deal with the additional unknown and equation resulting from this way of proceeding (see for instance [21]). In the framework of the present DDM, the additional unknown and equation are considered like the unknowns and equations corresponding to a cross point. In this way, this condition can be handled in a simple and natural manner.

The solution of direct problem (1.3) was carried out by a standard Uzawa algorithm, that is, by solving its Schur complement associated with the pressure by a conjugate gradient method (cf., e.g., [8]). The results for several unstructured meshes of $\Omega$ are given in Table 1 .

We have denoted by $\operatorname{Err}(\mathbf{u})$ and $\operatorname{Err}(p)$ the relative errors on the velocity and the pressure in respectively the $H^{1}$ - and the $L^{2}$-norm resulting from the approximation of the exact by the finite element solution. Domain $\Omega$ was decomposed into nine non-overlapping subdomains as this is depicted in Figure 4 to prepare the solution of (1.3) by the mixed DDM developed above and an adaptation of a FETI-DP method to the present context.

\subsection{Validation of the mixed DDM}

We have used two iterative procedures to deal with the above mixed DDM. The first one is the most straightforward, and consists in the relaxation iterative method with $\theta=1 / 2$. The second one is based on the Krylov method GMRES $(m)$ [36] where the integer $m$ indicates the dimension of the Krylov space used, usually referred to as the restart. We used GMRES(15) and GMRES(50) to test the behaviour of the DDM relatively to a small and a larger restart. In all cases, we have taken $\lambda=1$, zero as an initial guess and a reduction of the norm of the initial residual by $10^{-6}$.

The plot given in Figure 5 shows the decay of the residuals of the interface problem on $\Phi$ and $\psi$. As expected, GMRES(50) is the more efficient method followed by GMRES(15), the less successful being the relaxation scheme which stagnates after approximately 80 iterations. Table 2 reports the values of the highest and the lowest eigenvalue in magnitude of the matrix corresponding to the relaxation solver. We can thus directly get an estimate for the condition number $\kappa_{d}$ of the system related to the interface unknowns $\Phi$ and $\psi$.

This is in agreement with the above theoretical results ensuring that the successive approximation process converges. It is also worth mentioning that these values confirm the fact that the contraction constant depends on the mesh and more precisely that it tends to 1 as the mesh size $h \rightarrow 0$. Hence we recover a well-known feature of this kind of mixed DDM: the largest part of the error, that is, the one brought by the first spectral components of the latter, is rapidly damped by the relaxation solver which has some difficulties reducing the remaining part 


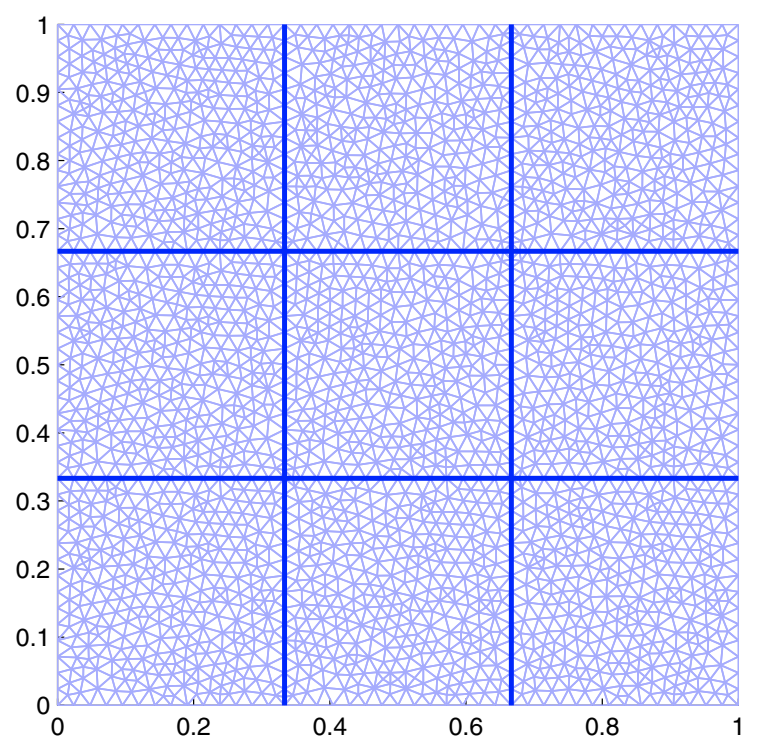

Figure 4. Partition of the domain into nine subdomains.

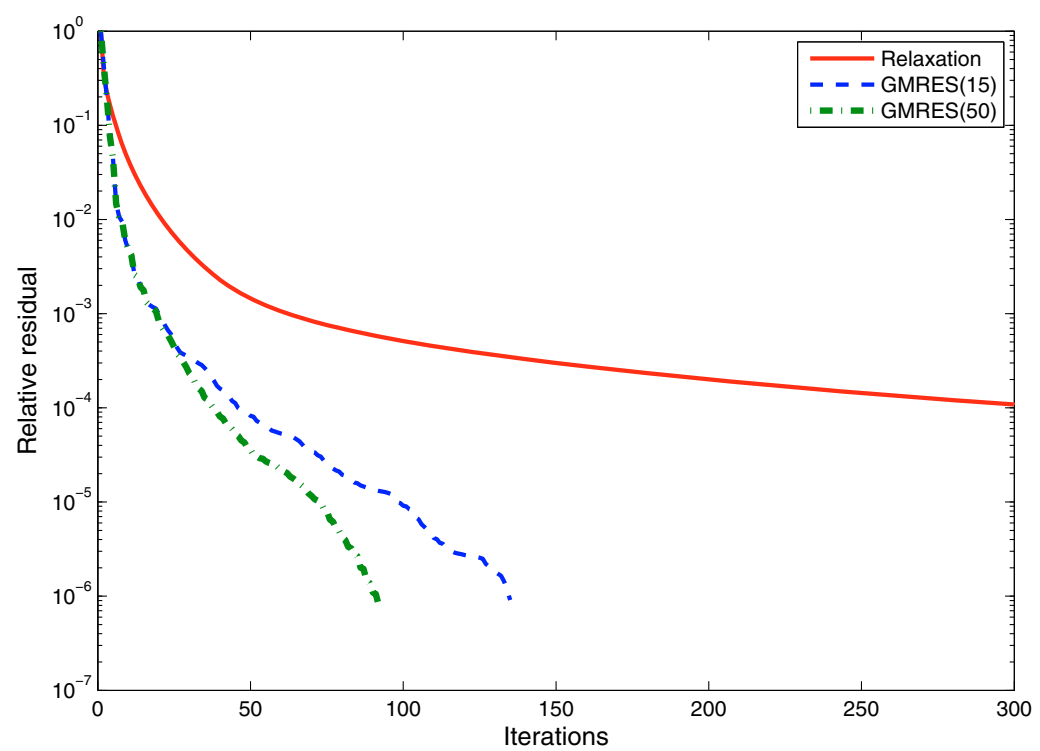

Figure 5. Decay of the residuals related to the three iterative solvers.

of the error corresponding to the highly oscillating components. For an investigation on this issue concerning the Helmholtz equation see, e.g., $[5,6]$.

Figure 6 depicts the decay of the relative errors for the velocity and the pressure, in $H^{1}(\Omega)$ and $L^{2}(\Omega)$ norms respectively. The errors are considered relatively to the velocity and the pressure obtained by solving discrete problem (1.3) by the direct method. In the plot, we have reported the errors inherent in the finite element 
TABLE 2. Smallest, highest eigenvalue in magnitude and condition number of the iteration matrix of the relaxation method.

\begin{tabular}{|c|c|c|c|}
\hline$h$ & $\lambda_{\max }$ & $\lambda_{\min }$ & $\kappa_{d}=\frac{\left|1-\lambda_{\min }\right|}{\left|1-\lambda_{\max }\right|}=10^{4} *$ \\
\hline $1 / 10$ & 0.9979 & -0.5 & 0.7 \\
\hline $1 / 20$ & 0.9980 & -0.5 & 0.7 \\
\hline $1 / 30$ & 0.9991 & -0.5 & 1.7 \\
\hline $1 / 40$ & 0.9991 & -0.5 & 1.7 \\
\hline $1 / 50$ & 0.9991 & -0.5 & 1.7 \\
\hline $1 / 60$ & 0.9991 & -0.5 & 1.7 \\
\hline
\end{tabular}

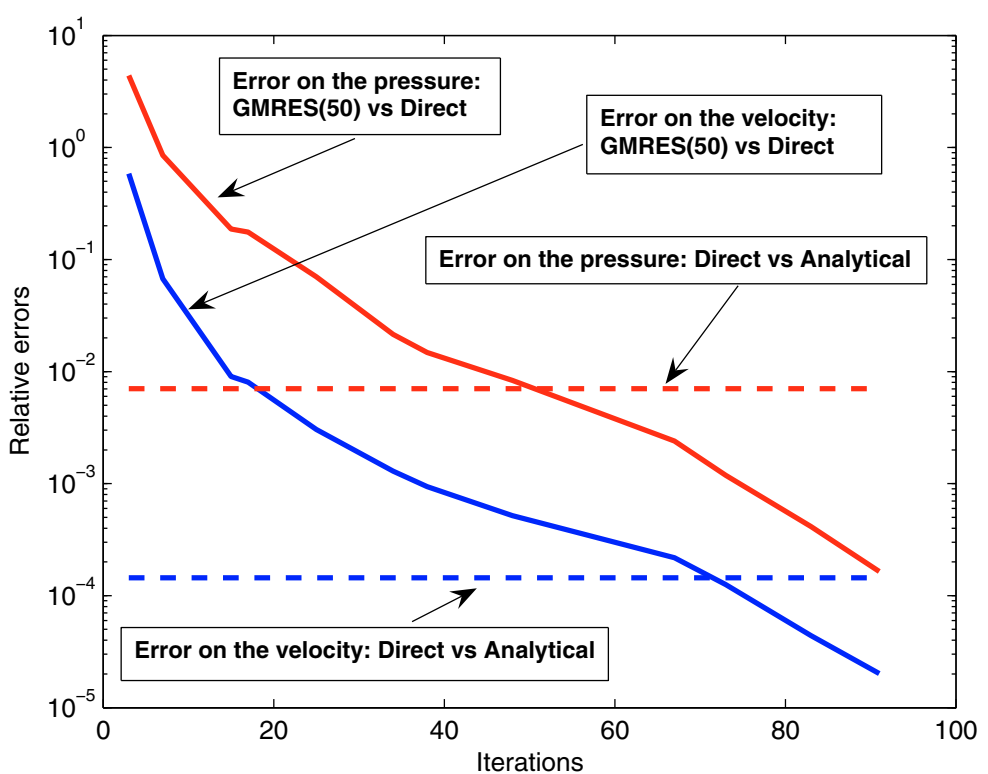

FiguRE 6 . Relative errors on the velocity and the pressure.

scheme resulting from the approximation of the analytical velocity and pressure by those related to the direct solution of (1.3). This gives an indication on the number of iterations which have really an impact on the overall accuracy of the numerical simulation. Also it is worth noting that, surprisingly enough, for the DDM as well as for the finite element scheme, the velocity converges faster than the pressure toward their respective limits.

Figure 7 depicts the pressure obtained at some iterations performed by the iterative solver GMRES(50). However, to get a better insight on the convergence of the DDM, we have plotted in Figure 8 the pressure for $y=1 / 3$ and for $0 \leq x \leq 1$ obtained after various iterations as well as its analytical value given by $p(x, 1 / 3)=-(x-1 / 2) / 6$.

This plot clearly indicates that, beyond 45 iterations, the iterative solver no longer improves the quality of the results.

\subsection{Comparison of the mixed DDM with a standard FETI-DP method}

We choose to apply the FETI-DP technique to the discrete problem (1.3) in a formal way as if it is a usual elliptic coercive problem like the ones arising in the finite element discretizations of static displacement formulations of structural mechanics problems (see for instance [18]). Such a way of proceeding does not 

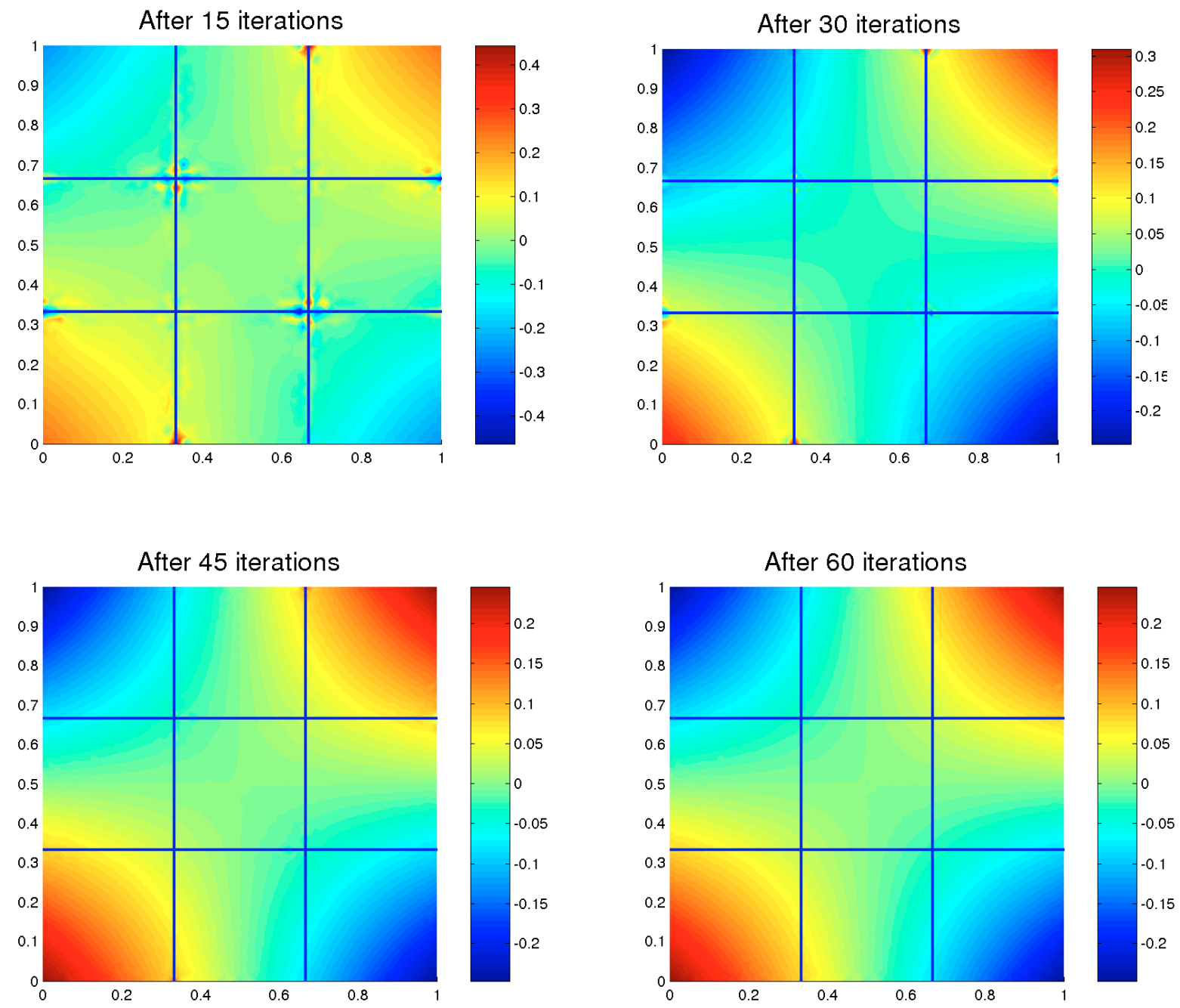

FiguRE 7. Intermediate pressure solution.

seem to have been considered for continuous pressure finite element approximations of the Stokes system. For instance, references $[22,23,26]$ used FETI-DP solvers only in the framework of finite element approximations of the pressure which are discontinuous at the interfaces of the elements. When dealing with a finite element scheme involving continuous pressures in this context as in [9], this continuity requirement is omitted at the interfaces of the subdomains involved in the domain decomposition procedure.

The adaptation of the FETI-DP method which is considered here uses almost the same framework as the previous mixed DDM, that is, we keep the same strong continuity requirements embodied by the spaces $X_{B}^{h}$ and $M_{B}^{h}$ at the cross points, but make use of only one unknown by interface instead of two. The interface unknowns and their associated test functions are thus running into the following subspace of $W^{h} \times Y^{h}$

$$
\left(W^{h} \times Y^{h}\right)_{s}=\left\{(\Phi, \psi) \in W^{h} \times Y^{h} ; \Phi_{i j}=\Phi_{j i} \text { and } \psi_{i j}=\psi_{j i}\right\}
$$




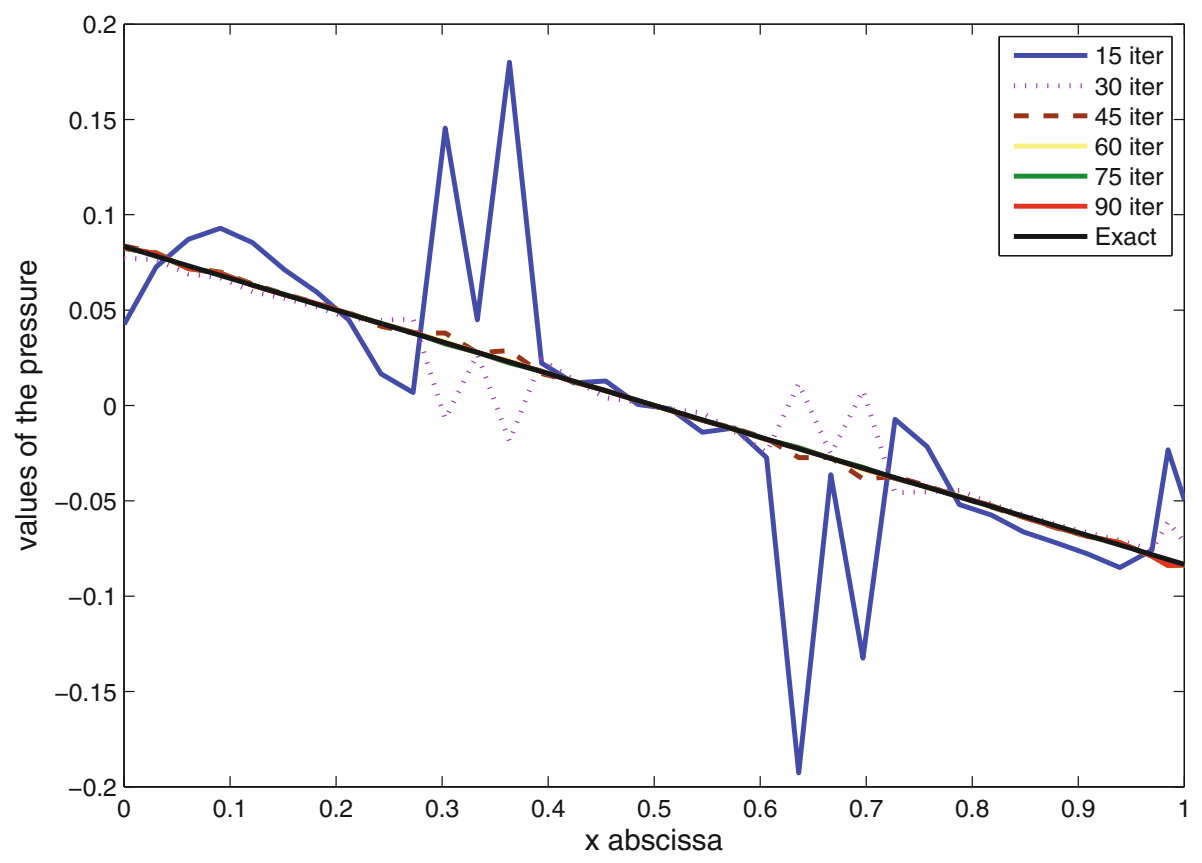

FiguRE 8. Exact and approximate values of the pressure at $y=1 / 3$. (Figure in color available online at www.esaim-m2an.org.)

We can then express equivalently the discrete problem (1.3) as follows

$$
\left\{\begin{aligned}
&(\mathbf{u}, p) \in X_{B}^{h} \times\left(M_{B}^{h} \cap L_{0}^{2}(\Omega)\right),(\Phi, \psi) \in\left(W^{h} \times Y^{h}\right)_{s}, \\
&\left\{\begin{array}{l}
i=1, \ldots, N \\
a_{i}\left(\mathbf{u}_{i}, \mathbf{v}_{i}\right)+b_{i}\left(\mathbf{v}_{i}, p_{i}\right)+a_{i}\left(\mathbf{u}_{c}, \mathbf{v}_{i}\right)+b_{i}\left(\mathbf{v}_{i}, p_{c}\right)+\sum_{j \in \Lambda_{i}} c_{i j}\left(\Phi_{i j}, \mathbf{v}_{i j}\right) \\
=\left(\mathbf{f}, \mathbf{v}_{i}\right)_{\Omega_{i}}, \\
b_{i}\left(\mathbf{u}_{i}, q_{i}\right)+b_{i}\left(\mathbf{u}_{c}, q_{i}\right)-\sum_{j \in \Lambda_{i}} d_{i j}\left(\psi_{i j}, q_{i j}\right)=0
\end{array}\right. \\
&\left\{\begin{array}{l}
\sum_{i=1}^{N}\left(a_{i}\left(\mathbf{u}_{i}, \mathbf{v}_{c}\right)+b_{i}\left(\mathbf{v}_{c}, p_{i}\right)\right)+a\left(\mathbf{u}_{c}, \mathbf{v}_{c}\right)+b\left(\mathbf{v}_{c}, p_{c}\right)=\left(\mathbf{f}, \mathbf{v}_{c}\right), \\
\sum_{i=1}^{N} b_{i}\left(\mathbf{u}_{i}, q_{c}\right)+b\left(\mathbf{u}_{c}, q_{c}\right)=0, \\
\sum_{i=1}^{N} \sum_{j \in \Lambda_{i}} c_{i j}\left(\Phi_{i j}^{\prime}, \mathbf{u}_{i j}\right)-d_{i j}\left(\psi_{i j}^{\prime}, p_{i j}\right)=0
\end{array}\right.
\end{aligned}\right.
$$

for all $(\mathbf{v}, q) \in X_{B}^{h} \times\left(M_{B}^{h} \cap L_{0}^{2}(\Omega)\right)$ and $\left(\Phi^{\prime}, \psi^{\prime}\right) \in\left(W^{h} \times Y^{h}\right)_{s}$. Here $c_{i j}$ and $d_{i j}$ are only the bilinear forms associated with boolean matrices corresponding to the matching conditions at the nodes ensuring that $\mathbf{u}$ and $p$ are continuous at the interfaces of the subdomains and therefore are such that $c_{i j}=-c_{j i}$ and $d_{i j}=-d_{j i}$. The zero mean-value condition on the global pressure is prescribed through a Lagrange multiplier which is joined to the degrees of freedom corresponding to the cross points as for the mixed DDM considered here.

As usual, the FETI-DP solver is implemented as an iterative procedure for solving the implicit linear system corresponding to the Schur complement of (3.2) associated with the interface unknowns. Clearly almost all the computing effort spent on performing the corresponding matrix-vector product is dedicated to the solution of (3.2) for a given set of interface unknowns $\Phi$ and $\psi$. This is done here also by solving the local problems twice and only once the problem for the cross points unknowns. We do no more detail this procedure here and refer to [20] where a general presentation of such a kind of methods for usual coercive problems is clearly developed 
TABLE 3. Convergence of the mixed DDM and the FETI-DP method.

\begin{tabular}{|c|c|c|c|c|c|c|}
\hline & \multicolumn{2}{|c|}{ Iterations } & \multicolumn{2}{c|}{ Rel. Err. $\mathbf{u}=10^{-5} *$} & \multicolumn{2}{c|}{ Rel. Err. $p=10^{-4} *$} \\
\hline$h$ & Mixed & FETI & Mixed & FETI & Mixed & FETI \\
\hline $1 / 10$ & 48 & 111 & 0.7 & 0.4 & 1.7 & 0.8 \\
\hline $1 / 20$ & 80 & 366 & 1.5 & 1.4 & 2.2 & 1.2 \\
\hline $1 / 30$ & 85 & 675 & 1.5 & 2.0 & 1.7 & 1.0 \\
\hline $1 / 40$ & 91 & 479 & 2.0 & 2.0 & 1.6 & 1.5 \\
\hline $1 / 50$ & 99 & 939 & 1.7 & 3.0 & 2.4 & 2.2 \\
\hline $1 / 60$ & 104 & 625 & 2.6 & 3.3 & 3.1 & 1.2 \\
\hline
\end{tabular}

TABLE 4. FETI-DP method with a Dirichlet preconditioner.

\begin{tabular}{|c|c|c|c|}
\hline$h$ & Iterations & Rel. Err. u & Rel. Err. $p$ \\
\hline $1 / 10$ & 22 & $8.06 \times 10^{-6}$ & $7.94 \times 10^{-4}$ \\
\hline $1 / 20$ & 32 & $4.52 \times 10^{-5}$ & $4.52 \times 10^{-3}$ \\
\hline $1 / 30$ & 36 & $7.95 \times 10^{-5}$ & $7.91 \times 10^{-3}$ \\
\hline $1 / 40$ & 36 & $7.55 \times 10^{-5}$ & $7.06 \times 10^{-3}$ \\
\hline $1 / 50$ & 38 & $1.74 \times 10^{-5}$ & $1.73 \times 10^{-2}$ \\
\hline $1 / 60$ & 42 & $2.50 \times 10^{-5}$ & $2.53 \times 10^{-4}$ \\
\hline
\end{tabular}

TABLE 5. Convergence of preconditioned and non preconditioned versions of the mixed and FETI-DP DDMs.

\begin{tabular}{|c|c|c|c|c|}
\hline$h$ & Mixed & Prec. Mixed & FETI-DP & Prec. FETI-DP \\
\hline $1 / 10$ & 48 & 22 & 111 & 22 \\
\hline $1 / 20$ & 80 & 23 & 366 & 32 \\
\hline $1 / 30$ & 85 & 26 & 675 & 36 \\
\hline
\end{tabular}

but without the present treatment of cross points. A procedure, closer to the present one but without any specific treatment for the pressure since only finite element discontinuous pressures are considered there, can also be found in [26].

It is however well-established that FETI algorithms poorly converge when they are not coupled with a suitable preconditioning technique (see for instance $[20,26]$ ). For example, used in the same framework as the previous mixed DDM, that is, with a decomposition of $\Omega$ in nine subdomains, with GMRES(50) and as a stopping criterion a reduction of $10^{-6}$ of the initial residual, it is outperformed by the DDM introduced in this study as this is clearly reported in Table 3. The relative errors are computed for the $H^{1}$-norm for the velocity and the $L^{2}$-norm for the pressure; the nominal solution is obtained by the Uzawa method. It is worth mentioning that the two methods deliver the same accuracy for the same stopping criterion.

To construct a preconditioner, we mimic the procedure used for usual coercive problems once more and adapt the Dirichlet preconditioner to this case (see, e.g., [20]). Here also, the way to proceed is close to the one used in [26] for the velocity but in our case for both the two unknowns. Table 4 now accounts for the improvement resulting from the preconditioning procedure at the expense this once of a accuracy slightly worse specially of the pressure.

The preconditioned FETI-DP is clearly more efficient than the mixed DDM at the expense however of some reliability based on a robust theoretical background for the latter. However to make a comparison of the two methods on a similar basis, we have also mimicked the strip preconditioning technique used in the context of mixed DDM for coercive problems [20]. It would be too lengthy to describe the whole technique here. We just give the number of iterations used by the four methods for three mesh sizes to converge in Table 5 . 
This clearly indicates that the advantage of the mixed DDM is restored by using a suitable preconditioner. A more thorough study of the numerical properties of these approaches will be given in a forthcoming study.

\section{Concluding Remarks}

This paper mainly dealt with theoretical issues linked to the construction and convergence of non overlapping DDMs for solving the discrete problems resulting from the discretization of the Stokes system by a mixed finite element method. Even if the construction chiefly concerns the continuous-pressure methods, the same framework can handle the discontinuous-pressure case equally well. The theoretical conclusions have been validated by numerical experiments which have also demonstrated the efficiency of such approaches at the practical level, specially when the DDMs are combined with the use of a Krylov solver. A thorough numerical study of the behaviour of such a kind of DDMs including some preconditioning techniques for the interface problem will be presented in a forthcoming work.

\section{REFERENCES}

[1] M. Ainsworth and S. Sherwin, Domain decomposition preconditioners for $p$ and $h p$ finite element approximation of Stokes equations. Comput. Methods Appl. Mech. Eng. 175 (1999) 243-266.

[2] A. Bendali and Y. Boubendir, Méthodes de décomposition de domaine et éléments finis nodaux pour la résolution de l'équation d'Helmholtz. C. R. Acad. Sci. Paris Sér. I 339 (2004) 229-234.

[3] A. Bendali and Y. Boubendir, Non-overlapping domain decomposition method for a nodal finite element method. Numer. Math. 103 (2006) 515-537.

[4] M. Bercovier and M. Engelman, A finite element for the numerical solution of viscous incompressible flows. J. Comput. Phys. 30 (1979) 181-201.

[5] Y. Boubendir, Techniques de décompositions de domaine et méthode d'équations intégrales. Ph.D. Thesis, INSA, Toulouse (2002).

[6] Y. Boubendir, An analysis of the BEM-FEM non-overlapping domain decomposition method for a scattering problem. J. Comput. Appl. Math. 204 (2007) 282- 291.

[7] S.C. Brenner and L.R. Scott, The mathematical theory of finite element methods. Springer-Verlag, New York (2002).

[8] F. Brezzi and M. Fortin, Mixed and hybrid finite element methods. Springer-Verlag, New York (1991).

[9] C. Calgaro and J. Laminie, On the domain decomposition method for the Stokes Problem with continuous pressure. Numer. Methods Partial Differ. Equ. 16 (2000) 84-106.

[10] P.G. Ciarlet, The finite element method for elliptic problems. North-Holland, Amsterdam (1978).

[11] T. Chacón Rebollo and E. Chacón Vera, A non-overlapping domain decomposition method for the Stokes equations via a penalty term on the interface. C. R. Acad. Sci. Paris Sér. I 334 (2002) 221-226.

[12] T. Chacón Rebollo and E. Chacón Vera, Study of a non-overlapping domain decomposition method: Poisson and Stokes problems. Appl. Numer. Math. 48 (2004) 169-194.

[13] F. Collino, S. Ghanemi and P. Joly, Domain decomposition method for harmonic wave propagation: a general presentation. Comput. Methods Appl. Mech. Eng. 184 (2000) 171-211.

[14] B. Després, Domain decomposition method and the Helmholtz problem, in Mathematical and Numerical Aspect of Wave Propagation Phenomena, SIAM, Philadelphia (1991) 44-52.

[15] M. Discacciati, A. Quarteroni and A. Valli, Robin-Robin domain decomposition methods for the Stokes-Darcy Coupling. SIAM J. Numer. Anal. 45 (2007) 1246-1268.

[16] C. Ferhat and F.X. Roux, A method of finite element tearing and interconnecting and its parallel solution alghorithm. Int. J. Numer. Methods Eng. 32 (1991) 1205-1227.

[17] C. Ferhat, M. Lesoinne, P. Le Tallec, K. Pierson and D. Rixen, FETI-DP: a dual-primal unified FETI method-part I: A faster alternative to the two-level FETI method. Int. J. Numer. Meth. Engng. 50 (2001) 1523-1544.

[18] V. Girault and P.A. Raviart, Finite Element Methods For Navier-Stokes Equations. Springer-Verlag, Berlin-Heidelberg (1986).

[19] V. Girault, B. Rivière and M.F. Wheeler, A discontinuous Galerkin method with non-overlapping domain decomposition for the Stokes and Navier-Stokes problems. Math. Comp. 74 (2004) 53-84.

[20] P. Gosselet and C. Rey, Non-overlapping domain decomposition methods in structural mechanics. Arch. Comput. Meth. Engng. 13 (2006) 515-572.

[21] D.Rh. Gwynllyw and T.N. Phillips, On the enforcement of the zero mean pressure condition in the spectral element approximation of the Stokes Problem. Comput. Methods Appl. Mech. Eng. 195 (2006) 1027-1049.

[22] H.H. Kim and C. Lee, A Neumann-Dirichlet preconditioner for a FETI-DP formulation of the two dimensional Stokes problem with mortar methods. SIAM J. Sci. Comput. 28 (2006) 1133-1152. 
[23] H.H. Kim, C. Lee and E.-H. Park, A FETI-DP formulation for the Stokes problem without primal pressure components. SIAM J. Numer. Anal. 47 (2010) 4142-4162.

[24] A. Klawonn and L.F. Pavarino, Overlapping Schwarz methods for elasticity and Stokes problems. Comput. Methods Appl. Mech. Eng. 165 (1998) 233-245.

[25] P. Le Tallec and A. Patra, Non-overlapping domain decomposition methods for adaptive hp approximations for the Stokes problem with discontinuous pressure fields. Comput. Methods Appl. Mech. Eng. 145 (1997) 361-379.

[26] J. Li, A Dual-Primal FETI methods for incompressible Stokes equations. Numer. Math. 102 (2005) $257-275$.

[27] P.L. Lions, On the Schwarz alternating method III: A variant for non-overlapping subdomains, in Third International Symposium on Domain Decomposition Methods for Partial Differential Equation, SIAM, Philadelphia (1990) $202-223$.

[28] G. Lube, L. Müller and F.C. Otto, A nonoverlapping domain decomposition method for stabilised finite element approximations of the Oseen equations. J. Comput. Appl. Math. 132 (2001) 211-236.

[29] J. Mandel and R. Tezaur, On the convergence of a dual primal substructuring method. Numer. Math. 88 (2001) 543-558.

[30] L.D. Marini and A. Quarteroni, Relaxation procedure for domain decomposition methods using finite elements. Numer. Math. 55 (1989) 575-589.

[31] F.C. Otto and G. Lube, A nonoverlapping domain decomposition method for the Oseen equations. Math. Models Methods Appl. Sci. 8 (1998) 1091-1117.

[32] F.C. Otto, G. Lube and L. Müller, An iterative substructuring method for div-stable finite element approximation of the Oseen problem. Computing 67 (2001) 91-117.

[33] L.F. Pavarino and O.B. Widlund, Balancing Neumann-Neumann methods for incompressible Stokes equations. Commun. Pure Appl. Math. 55 (2002) 302-335.

[34] A. Quarteroni and A. Valli, Domain decomposition methods for partial differential equations. Oxford University Press Inc., New York (1999)

[35] E.M. Rønquist, Domain decomposition methods for the steady Navier-Stokes equations, in 11th International Conference on Domain Decomposition Methods (London, 1998), DDM.org, Augsburg (1999) 330-340.

[36] Y. Saad, Iterative Methods for Sparse Linear Systems. PWS Publishing Company, Boston (1996).

[37] B. Vereecke, H. Bavestrello and D. Dureisseix, An extension of the FETI domain decomposition method for incompressible and nearly incompressible problems. Comput. Methods Appl. Mech. Eng. 192 (2003) 3409-3429. 